\title{
Investigating the floristic diversity indices of plant species in district Charsadda, Khyber Pakhtunkhwa, Pakistan
}

\author{
Sulaiman Shah ${ }^{1}$, Yaseen Khan ${ }^{2 *}$, Shariat Ullah ${ }^{1}$, Tabassum Yaseen ${ }^{3}$, \\ Shakir Ullah ${ }^{4}$ and Mian Fazli Basit ${ }^{3}$
}

1. Department of Botany, University of Malakand, Chakdara Dir lower, 18800, Khyber Pakhtunkhwa-Pakistan

2. Key Laboratory of Plant Nutrition and Agri-environment in Northwest China, Ministry of Agriculture, College of Natural Resources and Environment, Northwest A\&F University, Yangling, Shanxi-China

3. Department of Botany, Bacha Khan University, Charsadda, 24420, Khyber Pakhtunkhwa-Pakistan

4. Key Laboratory of Plant Ecology, Northeast Forestry University, Harbin, 150040-China

*Corresponding author's email: Yaseenkhan3444@gmail.com

Citation

Sulaiman Shah, Yaseen Khan, Shariat Ullah, Tabassum Yaseen, Shakir Ullah and Mian Fazli Basit. Investigating the floristic diversity indices of plant species in district Charsadda, Khyber Pakhtunkhwa, Pakistan. Pure and Applied Biology. Vol. 10, Issue 3, pp566-580. http://dx.doi.org/10.19045/bspab.2021.100059

\begin{tabular}{llll}
\hline \hline Received: 01/03/2020 & Revised: 29/10/2020 & Accepted: 02/11/2020 & Online First: 19/11/2020 \\
\hline \hline
\end{tabular}

\section{Abstract}

The floristic study carried out during 2017-2019 revealed that, district Charsadda comprised total of 146 plant species belonging to 58 families and 127 genera. The leading families were Asteraceae with 14 species (9.58\%), Poaceae 12 species (8.21\%), Solanaceae 8 species (5.47\%) and Cucurbitaceae 7 species (4.79\%). Apiaceae, Brassicaceae, Lamiaceae, Moraceae, Papilionaceae contribute by 5 species each (3.42\%), Chenopodiaceae, Rosaceae having 4 species each (2.73\%), Amaranthaceae, Euphorbiaceae, Malvaceae, Polygonaceae, Pteridaceae contributed by 3 species each (2.05\%), Alliaceae, Arecaceae, Asclepiadaceae, Cyperaceae, Ebinaceae, Equisetaceae, Fabaceae, Genetaceae, Geraniaceae, Mimosaceae, Myrtaceae, Oleaceae, Pinaceae, Rhamnaceae, Rutaceae contributed by 2 species each $(1.36 \%)$. The rest of the 27 families contributed by 1 species each $(0.68 \%)$. The most dominant life form was therophytes having 66 species (45.20\%), Microphanerophytes 22 species (15.06\%), and Chaemophytes 18 species (12.32\%). Hemicryptophyte 14 species $(9.58 \%)$ Nanophanerophyes 10 species $(6.84 \%)$, Geophytes 9 species $(6.16 \%)$, Megaphanerophytes 4 species $(2.75 \%)$ followed by Mesophanerophytes having 2 species $(1.36 \%)$. Leaf size spectra of the flora showed that the most dominant leaf size class were microphyll having 54 species (36.98\%), nanophyll 32 species (21.91\%), mesophyll 29 species (19.86\%), leptophylls 17 species (11.64\%), megaphyll contributed by 8 species $(5.47 \%)$ followed by macrophyll which represent 5 species $(3.04 \%)$ and the one species is aphyllus. The dominant therophytic life form showed that the flora of the area is under severe anthropogenic activity. This study not only gives information about the flora of Charsadda but can provide a baseline for future studies and plantation of this area.

Keywords: Floristic diversity; Life forms; Leaf spectrum; District Charsadda

\section{Introduction}

A huge number of plant species are yet to be uncovered by the botanist. Therefore, the floristic report is the only source to get botanical information about the area and it may help in starting for detailed study [1].
The word floristic is derived from flora which means to list all types of plant species or plant taxa within specific geographical area [2]. To improve conservation stratagems for plants of any area, it is necessary to have a comprehensive floristic record of the area 
centred on collection and correct documentation [3]. Floristic study is a very important for ecological sustainability and conservation of plants for an area. Floristic research is one of the most effective method to manage proper and protection of plants [4]. The documentation of the local plants with the description of an area is very necessary, because it can provide information throughout the time about distribution, occupancy, growing season and species rigidity of the available plants [5]. Floristic study also provide information about new species in a specific area, so by that way we can also analysis about migrated plants species [5]. Furthermore, the documentation of the plant species of the specific area is play chief role in environmental science respect to climate change [6]. Respect to climate change, Life form spectrum may also provide information about plant species, population size and their distribution over the area [7]. The life forms of plants are different in every zone on the basis of altitude. Three types of climates can be seen on earth, which includes phanerophytic in tropic, therophytic in desert and hemicryptophytic in cold temperate zone
[8]. This system of grouping of life forms is broadly accepted and it's has been globally followed.

The present study was started to report the floristic composition and its ecological characteristics of district Charsadda. District Charsadda is located in the west of Khyber Pakhtunkhwa province of Pakistan. It is bounded by district Malakand on the north, district Mardan on the east, district Peshawar on the south and district Mohmand on the west. It is situated on Latitude of $34.150 \mathrm{~N}$ and Longitude of 71.730E (Fig. 1) [9]. The district cover an area of 996 square kilometres and it is divided into 2 tehsils and 46 Union Councils. It is situated 282 meters above sea level in elevation. The predominant language is Pashto, spoken natively by $99.4 \%$ of the total population [10]. Temperature is variable from place to place, the coldest month of year is January, in which the average temperature is $5-10{ }^{\circ} \mathrm{C}$, while June is the hottest month in which the temperature raised up to $44^{\circ} \mathrm{C}$. The average rainfall is $82 \mathrm{~mm}$ per year. The most precipitation fall in the month of august [11].

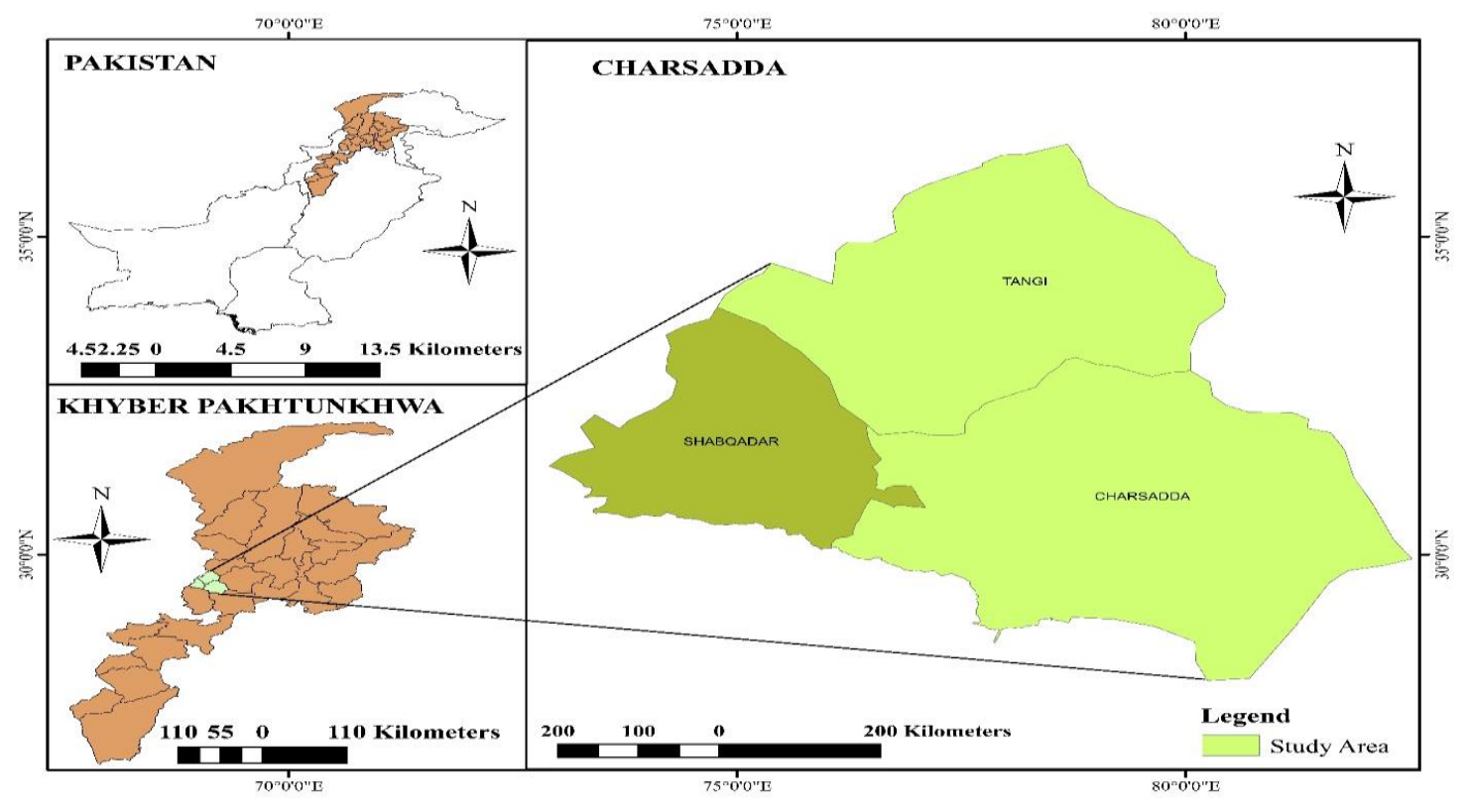


Figure 1. Map of district Charsadda (study area)

The main crops of the area are wheat, sugarcane, maize, tobacco, rice and several weeds [12]. Most of the area contains sandy, loamy and salty soil [13]. The vegetation can be found i.e herb, shrub, trees, dense vegetation are the maximum in number in this area, where the most common plant species are Xanthium strumarium, Cannabis sativa, Euphorbia helioscopia, Medicago denticulata, Withania somnifera [14]. In tress, Acacia nilotica, Ailanthus altisima, Broussonetia papyrifera, Morus alba, Morus nigra and Ziziphus jujube can be seen [15]. The present study might be helpful for the future researchers in the field of plant taxonomy related to district Charsadda. The purpose of this study was to explore the floristic composition, life form and leaf size spectra of district Charsadda.

\section{Materials and Methods}

Floristic study of district Charsadda was carried out during session of 2018-2019. Regular trips were arranged in various seasons (spring, summer, autumn and winter) to collect plant species. The locality, sub locality, vegetation, plant stage and leaf size were recorded after collection followed by [8]. The tools used during this research work were: Map of the area, notebook, pencil, plant presser, old newspaper, polythene bags, knife, compass and digital camera. The plant specimens were dried after identification. The plant specimens were submitted to herbarium, Department of botany, University of Malakand, Pakistan. The identification was carried out with the help of available literature [8-14]. The voucher specimens were deposited in the Herbarium of Centre of Plant Biodiversity, University of Malakand, Pakistan. The plant species were classified into different classes according to [8] as follows:

\section{Therophytes}

Annual seed bearing plants, which complete their life cycle in one year and overwinter; the unfavourable season by means of seeds or spores

\section{Geophytes}

Perennating buds located below the surface of soil including plants with deep rhizomes, bulbs, tubers and corms

\section{Hemicrytophytes}

Herbaceous perennial plants, in which the aerial portion of the plant dies at the end of the growing season, leaving a Perennating bud at or just beneath the ground surface

\section{Chamaeophytes}

Perennating buds located close to the ground surface (below the height of $25 \mathrm{~cm}$ ). The plants include herbaceous, low woody trailing, low stem succulents and cushion plants.

\section{Phanerophytes}

Most of the species are shrubby and tree, whose perennating buds are borne on aerial shoot reaching a height of at least $25 \mathrm{~cm}$ or more above the ground surface.

\section{Data analysis}

The data is analysed with Microsoft excel 2019.

\section{Results and Discussion Floristic and ecological attributes}

The flora of district Charsadda consisted of 146 plant species, which is belonging to 58 families. In this study, family Asteraceae was the dominating family having, 14 species including, Catharanthus roseus, Centaurea calcitropa, Hyphocharis radiate, Launaea nudicaulis, Onopordum acanthiuum, Silybum marianum, Taraxacum officinale and Xanthium strumarium. The subdominant families are Poaceae, Solanaceae and Cucurbitaceae with 7 species, followed by Apiaceae, Brassicaceae, Lamiaceae and Papilionaceae with 5 species. Chenopodiacea, Rosacea consisting 4 species, followed by Amaranthaceae, Euphorbiaceae, Malvaceae, Polygonaceae and Pteridaceae with 3 species. Alliaceae, Arecaceae, Asclepiadaceae, Cyperaceae, 
Ebinaceae, Equisetaceae, Fabaceae, Genetaceae, Geraniaceae, Mimocaceae, Myrtaceae, Oleaceae, Pinaceae, Rhamnaceae and Rutaceae with 2 species. Each and the rest of 27 Families having single specie (Fig. 2). Our finding is similar with Jan et al., Khan., Ali et al. and Qureshi et al. [16-19], in which the Asteraceae and Poaceae were leading families in their study work. Seasonal variation of vegetation shows that highest numbers of species i.e. 126 species, were found in summer followed by spring i.e. 108 species and autumn 73 species. While, compere to summer, less number of species can be seen in winter season i.e. 64 species (Fig. 3). Most of the herbaceous flora has varied distribution pattern in different seasons. The summer and spring flora is high in number than autumn and winter [20-22].

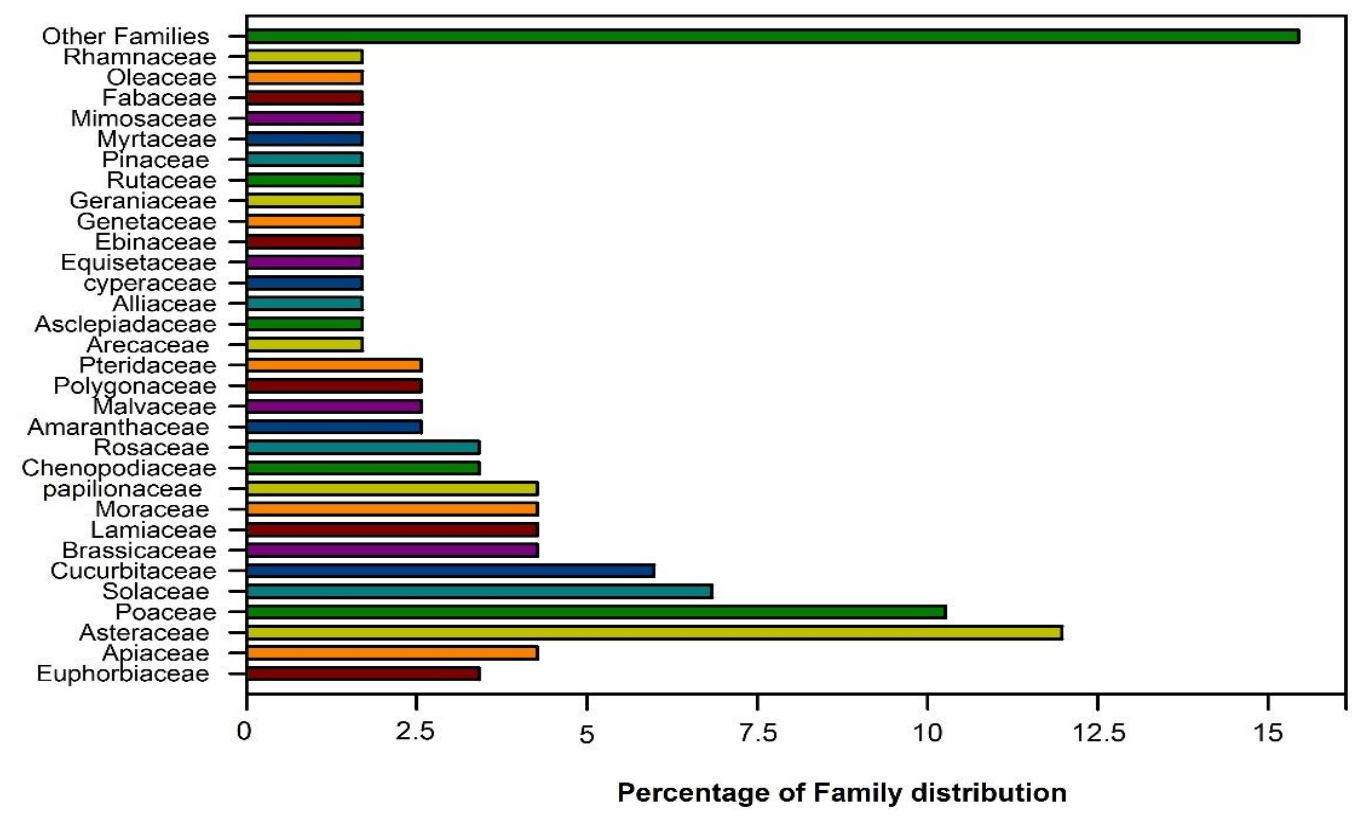

Figure 2. Plant families' percentage of vegetation conclusion

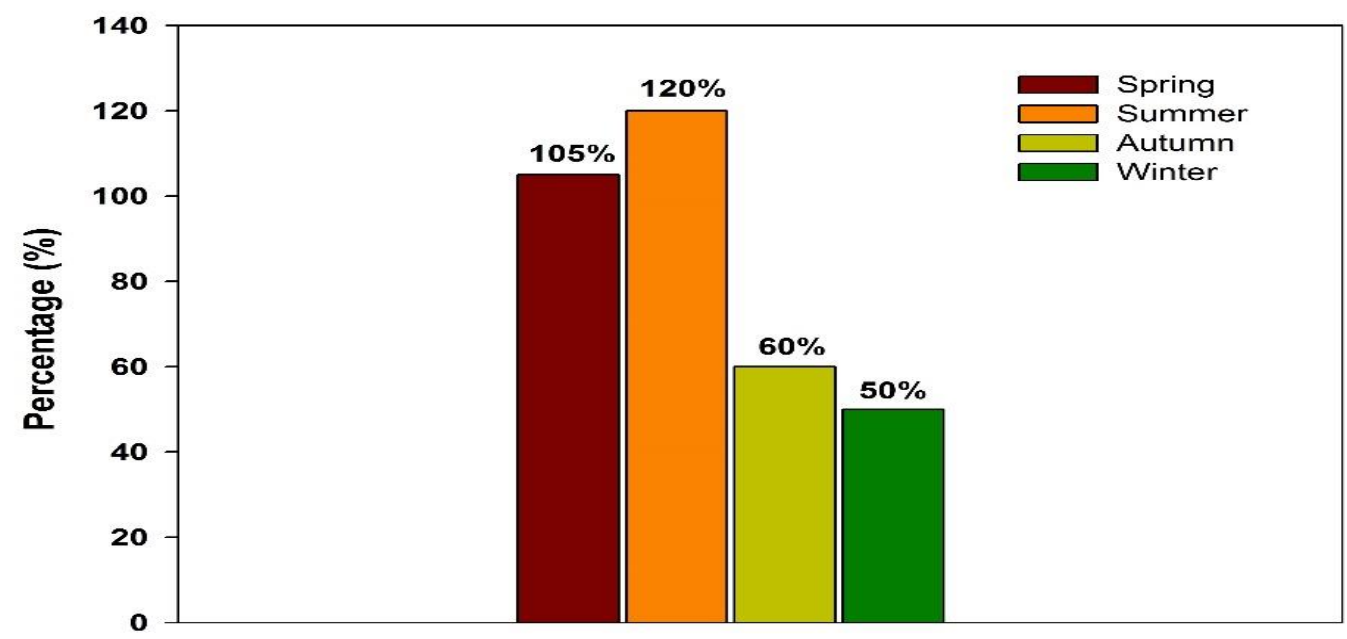

Percentage of number of species in different session 
Figure 3. Percentage of number of species in different season

Biological spectrum

Raunkiaer (1934) proposed the term "Biological Spectrum" to express both lifeform and distribution of flora and the phytoclimate under which the prevailing lifeforms evolved. The life-form study is thus an important part of vegetation description, ranking next to floristic composition. This study is an important part of the vegetation description, ranking next to floristic composition [23]. Life form and leaf size spectra indicate a climatic and human disturbance of a particular area [24]. The life form and leaf size spectra are beneficial attributes that have been broadly used in vegetation description. Furthermore, it is traditionally being used to describe world vegetation types at the community level [8]. The life form differences in various societies make up the basis of their structure. Different classification of the life forms there, but among them, Raunkiaer system is used most. Plants are divided into six main groups: Phanerophyte, Chamaephyte, Hemicryptophyte, Cryptophyte, Therophyte, and Epiphyte [25]. The percentage of life form was calculated as follows:

$\%$ Life-form $=$ Numbers of species in any life form $\times 100$ Total number of species of all life forms

All the plant species are classified into life forms and their ratio is expressed in number or percentage [26]. Furthermore, the biological spectrum was formed which showed that therophytes (66 spp., 45.20\%), microphanerophytes (22 spp., 15.06\%) (Fig. 4), chamaephytes (18 spp., 12.32\%), hemicryptophytes (14 spp., $9.58 \%)$, nanophanerophytes (10 spp., $18.5 \%)$, geophytes $\quad(9 \quad$ spp., $\quad 6.16 \%)$, megaphanerophytes (4 spp., $2.75 \%)$, mesophanerophytes (2 spp., 1.36\%) and parasite ( $1 \mathrm{spp} ., 0.68 \%$ ) had occurrence in the studied area (Table 1). The therophytes and chaemophytes dominancy showed that our study is similar to Durrani et al. Cain et al. Cain \& Castro [27-30] in which the therophytes, chaemophytes, hemicryptophytes were in dominant phase. The results of seasonal variation in different life classes revealed that in spring the therophytes (38 spp., 35.51\%) were dominant followed by microphanerophytes (22 spp., $20.56 \%$ ), chaemophytes (14 spp., 13.08\%), nanophanerophytes (10 spp., 9.34\%) and geophytes (9 spp., 8.41\%). Similar to spring, the dominant in summer the maximum numbers of species (52 spp., 41.6\%) were therophytes followed by Microphanerophytes (22 spp., 17.6\%), chaemophytes and hemicryptophytes (14 spp., $11.2 \%), \quad$ followed by nanophanerophytes (10 spp., 8\%), while in autumn, the dominant species were microphanerophytes (22 spp., 30.55\%) followed by therophytes (15 spp., 20.83\%), nanophanerophytes (10 spp., 13.88\%), chameophyes (9 spp., 12.5\%). The winter is the same as autumn the dominant species were microphaneophytes (22 spp., 34.37\%) followed by therophytes (11 spp., 17.18\%), nanophaneophytes (10 spp., 15.62\%), chaemophytes (9 spp., 14.06\%) (Fig. 5). Our result is similar regarding the dominancy of therophytes in spring and summer with Saxina et al. [31]. 

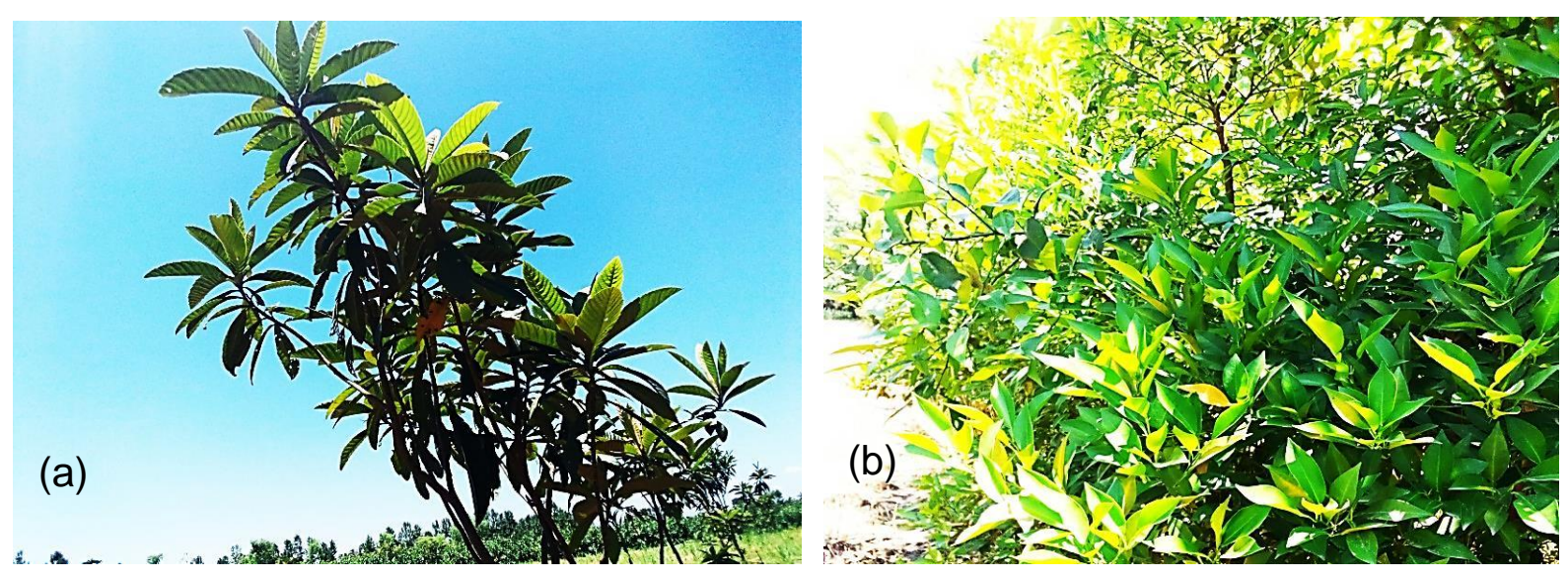

Figure 4(a,b). Microphanerophytes (MP)

Table 1. Seasonal variation in Life form spectra of vegetation of District Charsadda

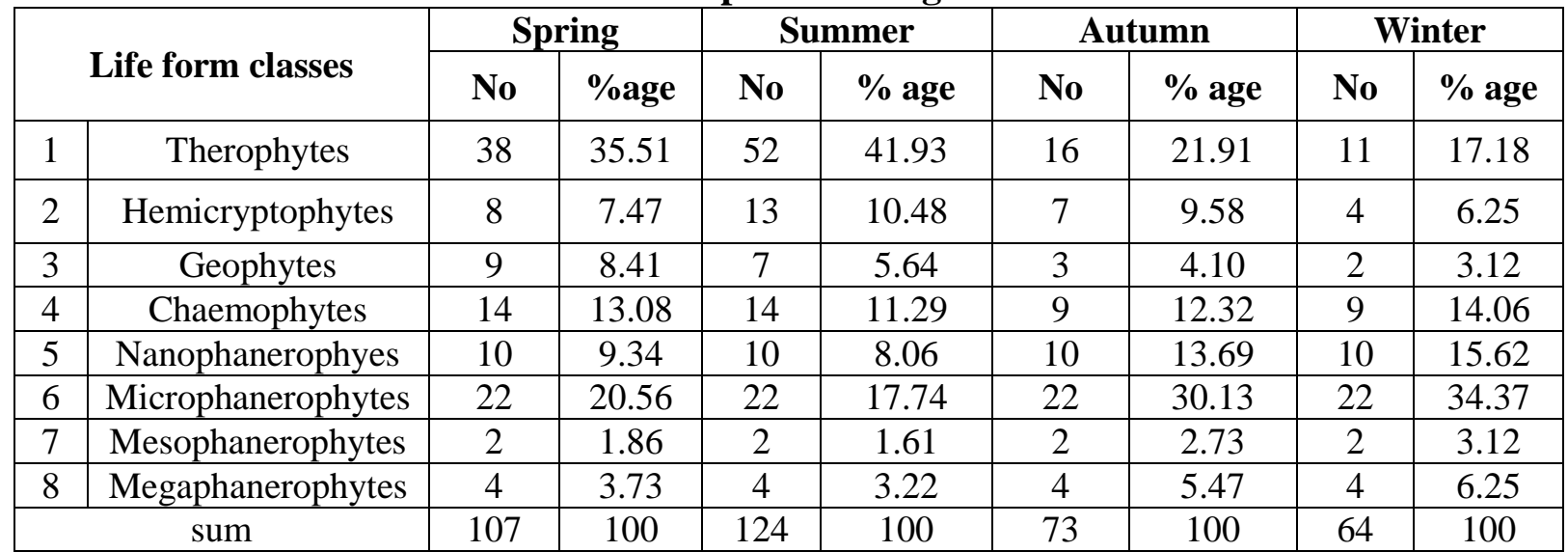

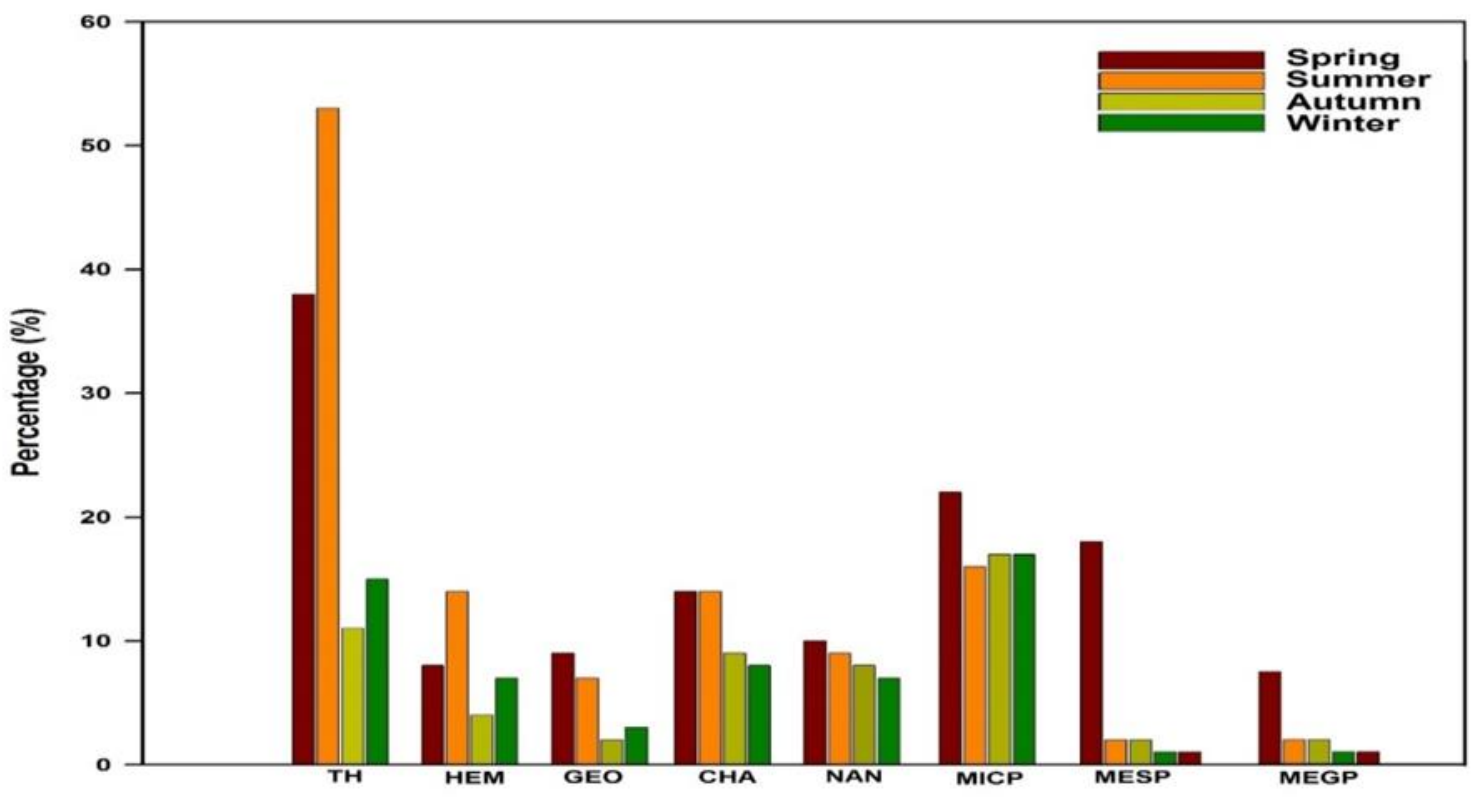

Figure 5. Seasonal variation in life form of vegetation 


\section{Leaf spectrum}

Life form and leaf size spectra indicate climatic and creature fracas of a particular area [24]. Leaf size classes have been found to be very useful for plant associations. Leaf size plays an important role in the physiological processes of plant and plant community in any area. The plant is also classified on the basis of leaf sizes [32]. In our study the leaf area of the species was calculated according to Ilyas et al. [24]. In our study, the dominant plant as regards leaf spectra was microphylls (54 spp., 36.98\%) followed by nanophylls (32 spp., 21.91\%), mesophylls (29 spp., $19.86 \%$ ), leptophylls (17 spp., 11.64\%), megaphylls (8 spp.,
$5.47 \%$ ) and macrophyll (5 spp., 3.04\%). While aphyllus represent only 1 specie (Fig. 5, 6, $7 \&$ 9; Table $2 \& 3$ ). Our present findings agreed with [33-42], in which microphylls and nanophylls were the dominant leaf-size classes.

\section{Habitat}

Habit is the general appearance, growth form and architecture of the plant species. In our study habit of species showed discrepancy, the main class was herbs having 95 species $(65.06 \%)$, followed by tree and shrub with 32 species $(21.91 \%)$ and 19 species $(13.01 \%)$ respectively. Our result showed low percentage of shrubs and trees species, which indicate severe deforestation in the area.

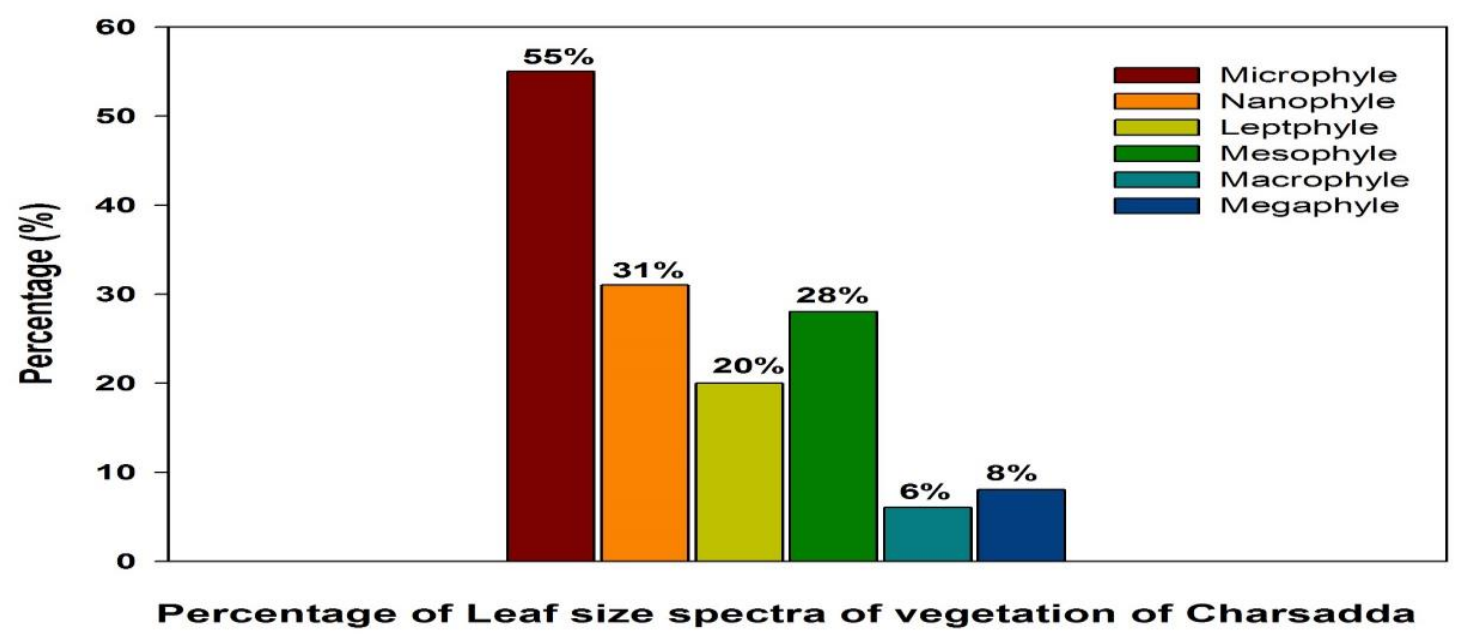

Figure 6. Percentage of Leaf size spectra of vegetation of district Charsadda

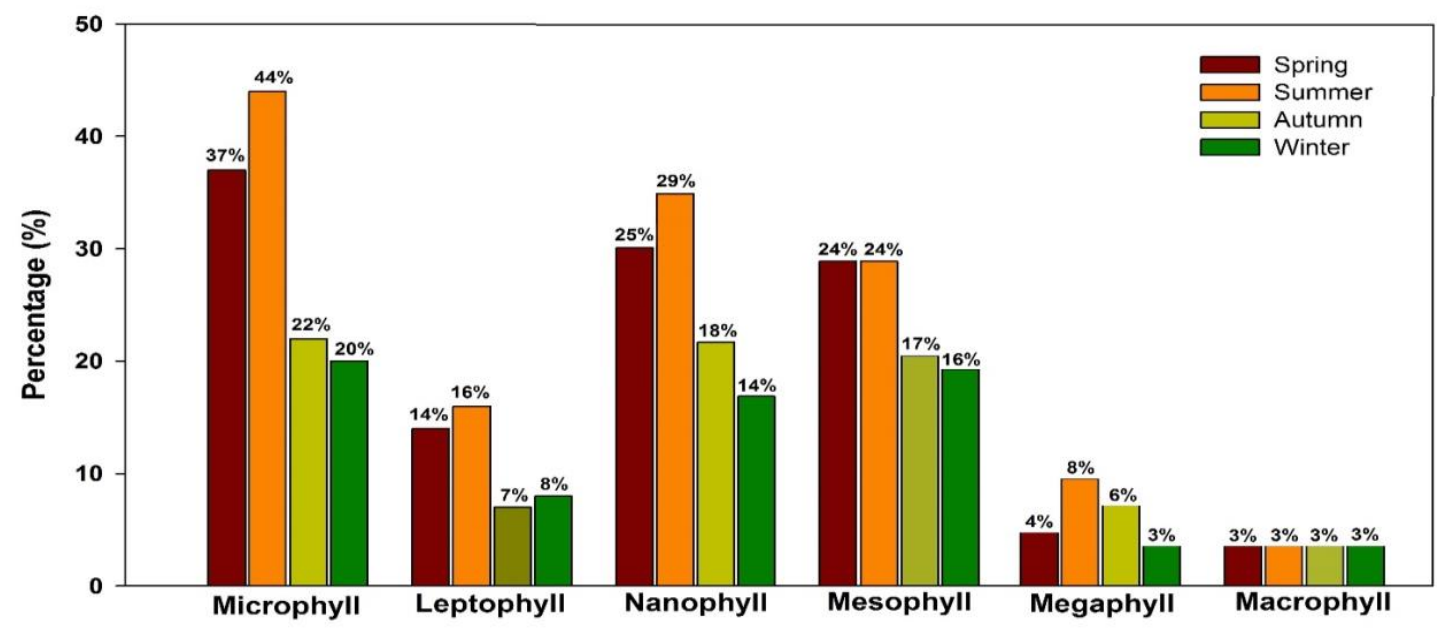

Figure 7. Percentage of Leaf size spectra in different season of district Charsadda 


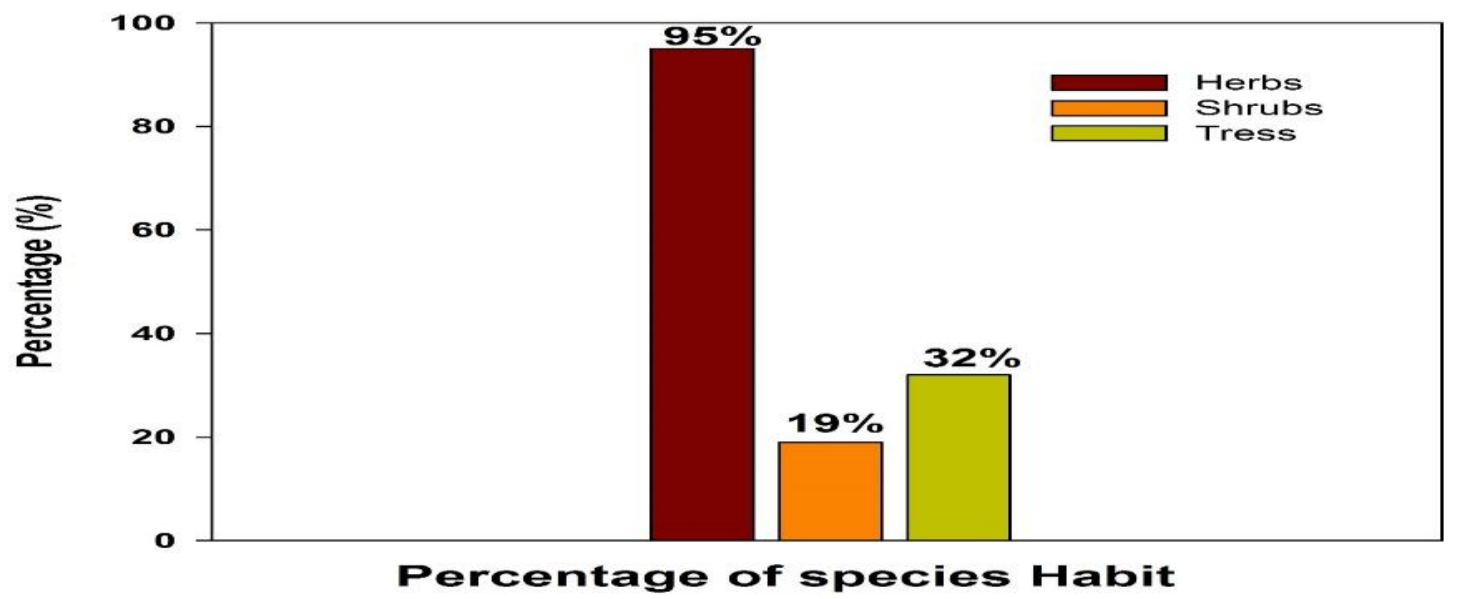

Figure 8. Percentage of species Habit

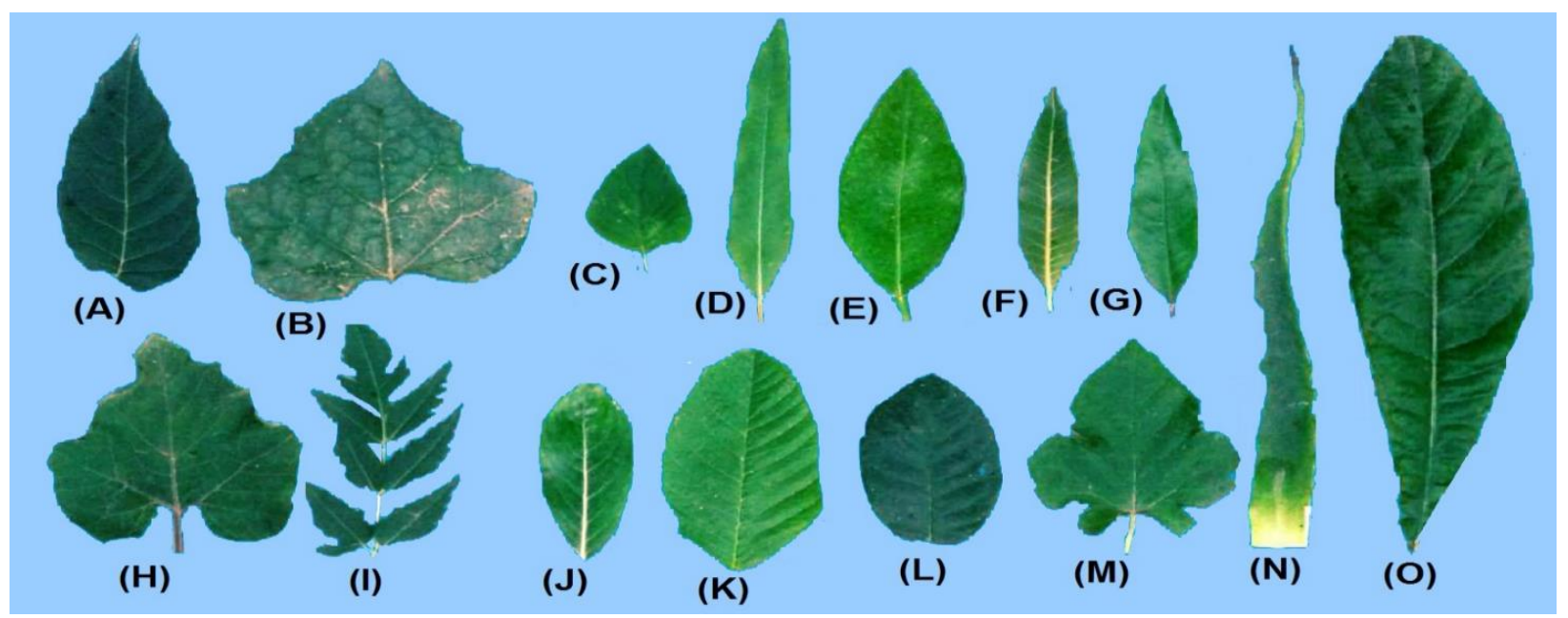

$\mathrm{A}=$ Mic, $\mathrm{B}=\mathrm{Mg}, \mathrm{C}=\mathrm{Nan}, \mathrm{D}=\mathrm{Mic}, \mathrm{E}=\mathrm{Mic}, \mathrm{F}=\mathrm{Mac}, \mathrm{G}=\mathrm{Nan}, \mathrm{H}=$ Meso, $\mathrm{I}=$ Nano, J= Meso, K= Mic, L= Meso, M= Nano, $\mathrm{N}=$ Mic, $\mathrm{O}=\mathrm{Mac}, \mathrm{P}=$ Meso

Figure 9. Leaf types in selected area

Table 2. Leaf size Spectra in a different type of Season of District Charsadda

\begin{tabular}{|c|c|c|c|c|c|c|c|c|c|}
\hline \multirow{2}{*}{\multicolumn{2}{|c|}{ Leaf size classes }} & \multicolumn{2}{|c|}{ Spring } & \multicolumn{2}{|c|}{ Summer } & \multicolumn{2}{|c|}{ Autumn } & \multicolumn{2}{|c|}{ Winter } \\
\hline & & \multirow{2}{*}{\begin{tabular}{|c|} 
No \\
37 \\
\end{tabular}} & \multirow{2}{*}{$\begin{array}{l}\text { \%age } \\
34.57\end{array}$} & \multirow{2}{*}{\begin{tabular}{|c|} 
No \\
44 \\
\end{tabular}} & \multirow{2}{*}{$\begin{array}{l}\text { \%age } \\
35.48\end{array}$} & \multirow{2}{*}{\begin{tabular}{|c|} 
No \\
22
\end{tabular}} & \multirow{2}{*}{$\begin{array}{l}\text { \% age } \\
30.13 \\
\end{array}$} & \multirow{2}{*}{\begin{tabular}{|l|} 
No \\
20
\end{tabular}} & \multirow{2}{*}{$\begin{array}{l}\text { \%age } \\
31.25\end{array}$} \\
\hline 1 & Microphyll & & & & & & & & \\
\hline 2 & Leptophyll & 14 & 13.08 & 16 & 12.90 & 7 & 9.58 & 8 & 12.5 \\
\hline 3 & Nanophyll & 25 & 23.36 & 29 & 23.38 & 18 & 24.65 & 14 & 21.87 \\
\hline 4 & Mesophyll & 24 & 22.42 & 24 & 19.35 & 17 & 23.28 & 16 & 25 \\
\hline 5 & Megaphyll & 4 & 3.73 & 8 & 6.45 & 6 & 8.21 & 3 & 4.68 \\
\hline 6 & Macrophyll & 3 & 2.80 & 3 & 2.41 & 3 & 4.10 & 3 & 4.68 \\
\hline & sum & 107 & 100 & 124 & 100 & 73 & 100 & 64 & 100 \\
\hline
\end{tabular}


Table 3. Floristic Composition, life Form, leaf size spectrum, habitat condition and seasonal variation of vegetation of District Charsadda KP, Pakistan

\begin{tabular}{|c|c|c|c|c|c|c|c|c|c|c|}
\hline \multirow{2}{*}{\multicolumn{2}{|c|}{ Plant species }} & \multirow{2}{*}{ Family } & \multirow{2}{*}{ Local name } & \multirow{2}{*}{ Habit } & \multirow{2}{*}{$\begin{array}{c}\text { Leaf } \\
\text { size }\end{array}$} & \multirow{2}{*}{$\begin{array}{c}\text { Life } \\
\text { form }\end{array}$} & \multicolumn{4}{|c|}{ Seasnality } \\
\hline & & & & & & & Sp & $\mathrm{Sm}$ & $\mathbf{W}$ & $\mathbf{A U}$ \\
\hline 1 & Allium sativa $\mathrm{L}$. & Alliaceae & Ooga & Herb & $\operatorname{mic}$ & Th & + & - & - & - \\
\hline 2 & Allium cepa L. & Alliaceae & Piyaz & Herb & mes & Geo & + & - & - & - \\
\hline 3 & Amaranthus viridus L. & Amaranthaceae & Ghanhar & Herb & Nan & $\mathrm{Th}$ & - & + & - & - \\
\hline 4 & Achyranthus aspera L. & Amaranthaceae & Not known & Herb & mic & Hem & - & + & - & + \\
\hline 5 & Alternanthera sessilis (L.) $\mathrm{RBr}$ exdc & Amaranthaceae & Not known & Herb & mic & $\mathrm{Th}$ & - & + & - & - \\
\hline 6 & Mangifera indica L. & Anacardiaceae & Aam & Tree & mes & Mesp & + & + & + & + \\
\hline 7 & Coriandrum sativm L. & Apiaceae & Danya & Herb & Lep & Th & + & + & - & - \\
\hline 8 & Capsicum fruitescens L. & Apiaceae & Tour mrach & Tree & mic & $\mathrm{Mp}$ & + & + & + & + \\
\hline 9 & Ammi visnaga (L.) Lam. & Apiaceae & sperkaye & Shrub & Lep & Cha & + & + & + & + \\
\hline 10 & Daucus carrota L. & Apiaceae & Ghajar & Herb & Mes & Geo & + & - & + & - \\
\hline 11 & Foeniculum vulgare mill. & Apiaceae & Unknown & Herb & Nan & Th & - & + & - & + \\
\hline 12 & Nannorrhops ritchiana (Griff.) Aitch & Arecaceae & Not known & Tree & $\mathrm{Mg}$ & $\mathrm{Mp}$ & + & + & + & + \\
\hline 13 & Phoenix dactylifera L. & Arecaceae & Kajora & Tree & $\mathrm{Mg}$ & $\mathrm{Mp}$ & + & + & + & + \\
\hline 14 & Calotropis procera (Aiton)w.t & Asclepiadaceae & $\begin{array}{c}\text { Speen } \\
\text { ponakay }\end{array}$ & Shrub & Mes & Cha & + & + & + & + \\
\hline 15 & Carulluma tuberculata N,E Brown & Asclepiadaceae & Pamankay & Herb & Nan & Th & + & + & + & + \\
\hline 16 & Agave sisalana perrineex Engelm & Asparagaceae & Unknown & Shrub & Meg & $\mathrm{Np}$ & + & + & + & + \\
\hline 17 & Hypocharis radiata Falk & Asteraceae & Shodapay & Herb & mes & Th & + & + & - & - \\
\hline 18 & Conyza stricta willd & Asteraceae & Kharboty & Herb & Mic & Cha & + & - & - & - \\
\hline 19 & Launaea nudicaulis (L) Hook .f. & Asteraceae & $\begin{array}{c}\text { Gora } \\
\text { shodapay }\end{array}$ & Herb & Mes & Th & + & - & + & - \\
\hline 20 & $\begin{array}{c}\text { Filago hardwaria (wall. exDc) } \\
\text { wagenitz }\end{array}$ & Asteraceae & Warkharay & Herb & Lep & Th & - & + & - & - \\
\hline 21 & Xanthium strumarium L. & Asteraceae & Gheshkay & Herb & $\mathrm{Mac}$ & Th & + & - & - & - \\
\hline 22 & Sonchus aspher L. & Asteraceae & $\begin{array}{c}\text { Ghta } \\
\text { shodapay }\end{array}$ & Herb & Nan & Hem & + & - & - & - \\
\hline 23 & Silybum marianum (L) Gaertn. & Asteraceae & Unknown & Herb & Mes & Th & + & + & - & - \\
\hline 24 & Catharanthus roseus (L.) G.Don & Asteraceae & Chaman gull & Herb & Mic & Th & - & + & - & - \\
\hline 25 & Calendula arvensis L. & Asteraceae & Zyar gully & Herb & Mic & $\mathrm{Th}$ & + & - & - & - \\
\hline 26 & Carthamus oxyacantha M.Bieb & Asteraceae & $\begin{array}{c}\text { Ghana } \\
\text { shodapay }\end{array}$ & Shrub & Mic & Th & - & + & - & - \\
\hline
\end{tabular}




\begin{tabular}{|c|c|c|c|c|c|c|c|c|c|c|}
\hline 27 & Centaurea calcitropa L. & Asteraceae & Unknown & Herb & Mes & Th & - & - & + & + \\
\hline 28 & Onopordum acanthium L. & Asteraceae & Ghana & Herb & Nan & Cha & + & + & - & - \\
\hline 29 & Parthenium hysterophorus L. & Asteraceae & Lewany botay & Herb & Mes & Th & + & + & - & + \\
\hline 30 & Taraxacum officinale weber & Asteraceae & $\begin{array}{l}\text { Zyargul } \\
\text { shodapy }\end{array}$ & Herb & Mic & Th & + & + & - & - \\
\hline 31 & Coronopus didymus (L) Sm & Brasicaceae & Sqaboty & Herb & Lep & $\mathrm{Th}$ & + & + & - & - \\
\hline 32 & Raphanus raphanistrum L. & Brasicaceae & Tapermoly & Herb & Mes & $\mathrm{Th}$ & + & + & - & - \\
\hline 33 & Eruca sativa Mill. & Brasicaceae & Teparge & Herb & Mes & Cha & + & + & - & - \\
\hline 34 & Capsella bursa-pastoris medic & Brasicaceae & $\begin{array}{c}\text { Tour } \\
\text { sharsham }\end{array}$ & Herb & Mic & Th & + & - & + & - \\
\hline 35 & Brassica compestris L. & Brasicaceae & Sharsham & Herb & Mic & Th & + & - & + & - \\
\hline 36 & Opuntia littoralis (englim.) & Cactaceae & Zuqam & Shrub & Lep & $\mathrm{Np}$ & + & + & + & + \\
\hline 37 & Cannabis sativa L. & Cannabaceae & Bang & Herb & Mic & Th & + & + & + & - \\
\hline 38 & Stellaria media (L.) cry & Cryopphyllaceae & Unknown & Herb & Nan & Th & + & + & - & - \\
\hline 39 & Chenopodium album L. & Chenopodiaceae & Spensag & Herb & Mic & Th & + & - & - & + \\
\hline 40 & Spinacea oleracea L. & Chenopodiaceae & Palak & Herb & Mic & $\mathrm{Th}$ & + & + & - & - \\
\hline 41 & Chenopodium murale L. & Chenopodiaceae & Sag & Herb & Lep & Th & - & + & - & - \\
\hline 42 & Kochia indica wight & Chenopodiaceae & Sqagaya & Shrub & Nan & Cha & + & + & + & + \\
\hline 43 & Convolvulus arvensis L. & Convolvulaceae & Unknown & Herb & Nan & Th & - & + & - & - \\
\hline 44 & Citrulus colocynthis (L) schrad & Cucurbitaceae & Unknown & Herb & Mic & Th & - & + & - & - \\
\hline 45 & Citrulus lanatus (thum.)mats & Cucurbitaceae & Hindwana & Herb & Mes & Th & - & + & - & - \\
\hline 46 & Cucurbita maxima Duchesne & Cucurbitaceae & Kado & Herb & $\mathrm{Mg}$ & $\mathrm{Th}$ & - & + & - & + \\
\hline 47 & Luffa cylinderica (L.) Roem & Cucurbitaceae & Tori & Herb & $\mathrm{Mg}$ & Th & - & + & - & + \\
\hline 48 & Momordica charantia L. & Cucurbitaceae & Karela & Herb & Mes & Th & - & + & - & + \\
\hline 49 & Cucumis melo L. & Cucurbitaceae & Harboza & Herb & Mac & Th & - & + & - & + \\
\hline 50 & Cucurbita pepo L. & Cucurbitaceae & Harbaza & Herb & $\mathrm{Mg}$ & Th & - & + & - & + \\
\hline 51 & Cuscuta reflexa Roxb & Cuscutaceae & Unknown & Herb & Lep & $\mathrm{P}$ & + & + & - & + \\
\hline 52 & Cyperus niveus Retz & Cyperaceae & Unknown & Herb & Nan & Hem & - & + & - & - \\
\hline 53 & Cyperus rotundus L. & Cyperaceae & Unknown & Herb & Nan & Th & - & + & - & - \\
\hline 54 & Diospyros kaki L. & Ebinaceae & Amlok & Tree & Mes & $\mathrm{Mp}$ & + & + & + & + \\
\hline 55 & Diospyros lotus L. & Ebinaceae & Amlok & Tree & Mic & megp & + & + & + & + \\
\hline 56 & Equisetum ramossimum Desf & Equisetaceae & Bandaky & Herb & Ap & Geo & + & + & - & + \\
\hline 57 & Equisetum arvensis L. & Equisetaceae & Bandaky & Herb & Lep & Hem & + & + & - & + \\
\hline 58 & Euphorbia helioscopia L. & Euphorbiaceae & Mandaro & Herb & Nan & Th & - & + & - & - \\
\hline 59 & Euphorbia prostrata Aiton & Euphorbiaceae & Unknown & Herb & Lep & Th & + & - & + & - \\
\hline
\end{tabular}




\begin{tabular}{|c|c|c|c|c|c|c|c|c|c|c|}
\hline 60 & Ricinus communis L. & Euphorbiaceae & Pomba & Shrub & Nan & Megp & + & + & + & + \\
\hline 61 & Medicago denticulata Willd & Fabaceae & Peshtaray & Herb & Nan & Th & + & - & - & - \\
\hline 62 & Trifolium alexandrium L. & Fabaceae & Riksha & Herb & Nan & $\mathrm{Th}$ & + & - & - & - \\
\hline 63 & Fumaric indica Pugsley & Fumariaceae & Unknown & Herb & Lep & Th & - & + & - & - \\
\hline 64 & Centaurium pulchellum (Sw.) Druce & Gentianaceae & Kargha mewa & Herb & Mic & Th & - & + & - & + \\
\hline 65 & Swertia ciliate (G .Don ) B.L Bur & Gentianaceae & Unknown & Herb & Mes & Th & - & + & - & + \\
\hline 66 & Erodium cicutarium $(\mathrm{L}) \mathrm{L}$, Heritex & Geraniaceae & Unknown & Herb & Mac & Cha & + & - & + & - \\
\hline 67 & Geranium wlinum sweet & Geraniaceae & Unknown & Herb & Mic & Hem & - & + & - & - \\
\hline 68 & Juglans regia L. & Juglandaceae & Ghoz & Tree & Mic & $\mathrm{Mp}$ & + & + & + & + \\
\hline 69 & Ocimum bascillicum L. & Lamiaceae & Kashmaly & Shrub & Nan & Cha & + & + & + & + \\
\hline 70 & Mentha arvensis L. & Lamiaceae & Venally & Herb & Nan & Geo & + & + & - & + \\
\hline 71 & Mentha sylvestris L. & Lamiaceae & Venally & Herb & Nan & Th & + & + & - & - \\
\hline 72 & Mentha longifolia L. & Lamiaceae & Venally & Herb & Mic & Geo & + & + & - & - \\
\hline 73 & Salvia lanata Roxb & Lamiaceae & Unknown & Herb & Mes & Th & - & + & + & + \\
\hline 74 & Malva neglecta Wallr & Malvaceae & Panderak & Herb & Mic & Th & + & + & + & - \\
\hline 75 & Ablemoschus esculentus L. & Malvaceae & Unknown & Herb & Mic & Th & - & + & - & - \\
\hline 76 & $\begin{array}{c}\text { Malvastrum coromandelianum (L.) } \\
\text { Garcke }\end{array}$ & Malvaceae & Ghanta boty & Herb & Mic & Th & + & - & - & - \\
\hline 77 & Melia azedarach L. & Meliaceae & Bakyana & Tree & Nan & $\mathrm{Mp}$ & + & + & + & + \\
\hline 78 & Ficus palmate Forssk & Moraceae & Waroki inzar & Tree & Mes & $\mathrm{Mp}$ & + & + & + & + \\
\hline 79 & Ficus carica L. & Moraceae & Ghat inzar & Tree & Mes & $\mathrm{Np}$ & + & + & + & + \\
\hline 80 & Morus alba L. & Moraceae & Speen tot & Tree & Mes & $\mathrm{Mp}$ & + & + & + & + \\
\hline 81 & Morus nigra L. & Moraceae & Tor tot & Tree & Mes & $\mathrm{Mp}$ & + & + & + & + \\
\hline 82 & Broussonetia papyrifera (L.) Vent & Moraceae & Shahtot & Tree & Mac & Megp & + & + & + & + \\
\hline 83 & Acacia nilotica (L.) Delile & Mimosaceae & Kikar & Tree & Lep & $\mathrm{Mp}$ & + & + & + & + \\
\hline 84 & Acacia fernesiana (L) willd & Mimosaceae & Ghana & Tree & Lep & $\mathrm{Mp}$ & + & + & + & + \\
\hline 85 & Eucalyptas lanceolatus -Honey & Myrtaceae & Lachi & Tree & Mic & Cha & + & + & + & + \\
\hline 86 & Psidium guajava L. & Myrtaceae & Amrod & Tree & Mes & $\mathrm{Mp}$ & + & + & + & + \\
\hline 87 & Olea ferruginea Royle & Oleaceae & Jaman & Tree & Mic & $\mathrm{Mp}$ & + & + & + & + \\
\hline 88 & Jasminum officinale L. & Oleaceae & Unknown & Tree & Nan & $\mathrm{Np}$ & + & + & + & + \\
\hline 89 & Oxalis corniculata L. & Oxalidaceae & Trewaky & Herb & Nan & Th & + & + & - & + \\
\hline 90 & Phaseolus vulgare L. & Papailionaceae & Unknown & Shrub & Mes & Cha & - & + & - & - \\
\hline 91 & Papaver somniferum L. & Papaveraceae & Bang & Herb & Mes & Th & + & + & - & - \\
\hline 92 & Abizia lebbeck (L.) Benth & Papilionaceae & Emli & Tree & Lep & $\mathrm{Mp}$ & + & + & + & + \\
\hline 93 & Pisum sativum L. & Papilionaceae & Matar & Herb & Mic & Th & + & - & - & - \\
\hline
\end{tabular}




\begin{tabular}{|c|c|c|c|c|c|c|c|c|c|c|}
\hline 94 & Astragulus hamosus (L) Boiss & Papilionaceae & Jamdar & Herb & Mic & Th & - & + & - & - \\
\hline 95 & Delbegia sesso Roxb. ExDc & Papilionaceae & Shawa & Tree & Nan & $\mathrm{Mp}$ & + & + & + & + \\
\hline 96 & Vicia hirsuta (L). Gray & Papilionaceae & Mompali & Herb & Nan & Th & + & + & - & - \\
\hline 97 & Pinus roxburghii Sargent & Pinaceae & Nakhtr & Tree & Lep & Mesp & + & + & + & + \\
\hline 98 & Pinus wellichiana A.B.Jackson & Pinaceae & Deyar & Tree & Lep & Megp & + & + & + & + \\
\hline 99 & Cynodon dictylon (L) Pers & Poaceae & Kabal & Herb & Nan & Hem & + & + & + & + \\
\hline 100 & Aristida adscensions L. & Poaceae & Wakha & Herb & Mic & Hem & - & + & - & - \\
\hline 101 & Bromus japonicus Houtt & Poaceae & Jaodar & Herb & Mic & Hem & - & + & - & - \\
\hline 102 & Cenchrus ciliaris L. & Poaceae & Kalak wakha & Herb & Lep & Hem & + & + & - & - \\
\hline 103 & Secale cereal L. & Poaceae & Warbashi & Herb & Mic & Th & - & + & + & - \\
\hline 104 & $\begin{array}{c}\text { Phragmites australis (cav) Trinex } \\
\text { steud }\end{array}$ & Poaceae & Makay & Shrub & Mes & Geo & + & + & - & - \\
\hline 105 & Echinochola colona L. & Poaceae & Koray & Herb & Nan & Hem & - & + & - & - \\
\hline 106 & Phalaris minor Retz & Poaceae & Gaya & Herb & Mic & Th & + & + & - & - \\
\hline 107 & Avena sativa $\mathrm{L}$. & Poaceae & Jaodar & Herb & Mic & Th & + & - & - & - \\
\hline 108 & Zea mays $\mathrm{L}$. & Poaceae & Jowar & Herb & $\mathrm{Mg}$ & Th & - & + & - & - \\
\hline 109 & Triticum estivum L. & Poaceae & Ghanam & Herb & Mic & Th & - & + & - & - \\
\hline 110 & Oryza sativa $\mathrm{L}$. & Poaceae & Roji & Herb & Mic & Geo & + & + & - & - \\
\hline 111 & Rumix hastatus L. & Polygonaceae & Shalkhy & Herb & Mes & Th & + & - & - & - \\
\hline 112 & $\begin{array}{l}\text { Homalocladium platycledum } \\
\text { (F.Muell). }\end{array}$ & Polygonaceae & Unknown & Shrub & Mes & Cha & + & + & + & + \\
\hline 113 & Polygonam baratum L. & Polygonaceae & Unknown & Herb & Mic & Cha & + & - & - & + \\
\hline 114 & Portulaca oleraceae L. & Portulaceae & Warkhary & Herb & Nan & Th & + & + & - & - \\
\hline 115 & Pteris vitata $\mathrm{L}$. & Ptridaceae & Unknown & Herb & Mic & Hem & + & + & + & + \\
\hline 116 & Pteris cretica $\mathrm{L}$ & Ptridaceae & Unknown & Herb & Mic & Hem & + & + & + & + \\
\hline 117 & Adiantum-capillus-veneris L. & Ptridaceae & Unknown & Herb & Nan & $\mathrm{Hem}$ & + & + & + & + \\
\hline 118 & Punica granatum $\mathrm{L}$. & Punicaeae & Anar & Tree & Nan & $\mathrm{Np}$ & + & + & + & + \\
\hline 119 & Rananculus muricatus L. & Rananculaceae & Jaghagha & Herb & Mic & Geo & + & + & - & - \\
\hline 121 & Zizphus mauritiana lam. & Rhamnaceae & Sara bera & Tree & Nan & $\mathrm{Mp}$ & + & + & + & + \\
\hline 122 & Rosa india $\mathrm{L}$. & Rosaceae & Gulab & Shrub & Mic & $\mathrm{Np}$ & + & + & + & + \\
\hline 123 & Rosa webiana $\mathrm{L}$. & Rosaceae & Gulab & Shrub & Mic & $\mathrm{Np}$ & + & + & + & + \\
\hline 124 & Eriobotrya japonica (Thunb) Lindl & Rosaceae & Loakat & Tree & Mac & $\mathrm{Mp}$ & + & + & + & + \\
\hline 125 & Prunus armeniaca $\mathrm{L}$ & Rosaceae & Khubany & Tree & Nan & $\mathrm{Mp}$ & + & + & + & + \\
\hline 126 & Galium aparine L. & Rubiaceae & Kotriboty & Herb & Lep & Th & + & + & - & - \\
\hline 127 & Citrus indica $\mathrm{L}$. & Rutaceae & Naranj & Shrub & Mic & Micp & + & + & + & + \\
\hline
\end{tabular}




\begin{tabular}{|c|c|c|c|c|c|c|c|c|c|c|}
\hline 128 & Zanthoxylum armatum Dc & Rutaceae & Unknown & Tree & Mic & $\mathrm{Np}$ & + & + & + & + \\
\hline 129 & Dodonea viscosa (L.) Jacq & Sapindaceae & Ghorasky & Tree & Nan & $\mathrm{Np}$ & + & + & + & + \\
\hline 130 & Manilkara zapota (L.) P.Royen & Sapotaceae & Cheko & Tree & Mic & $\mathrm{Mp}$ & + & + & + & + \\
\hline 131 & Verbascum Thapsus L. & Scrophulariaceae & Harghwagh & Herb & Meg & Th & + & + & - & - \\
\hline 132 & Ailanthus altissima (mill) swiingle & Simarubaceae & $\begin{array}{l}\text { Paramy } \\
\text { shandy }\end{array}$ & Tree & Mic & Mp & + & + & + & + \\
\hline 133 & Solanum nigram L. & Solanaceae & Kachmacho & Herb & Mic & $\mathrm{Th}$ & + & + & - & - \\
\hline 134 & Datura metel L. & Solanaceae & Batura & Shrub & Mic & $\mathrm{Th}$ & + & + & - & - \\
\hline 135 & Withania somnifera (L.) Dunal & Solanaceae & Batwa boty & Shrub & Mic & Cha & - & + & - & - \\
\hline 136 & Withania coagulans (stocks) Dunal & Solanaceae & Batwa boty & Shrub & Mic & Cha & - & + & - & - \\
\hline 137 & Solanum surrattense Burn .f & Solanaceae & Unknown & Herb & Nan & Hem & + & + & - & + \\
\hline 138 & Physalis minima L. & Solanaceae & Unknown & Herb & Mic & Cha & - & + & - & + \\
\hline 139 & Lycopersicun esculentum Mill & Solanaceae & Tamatar & Herb & Mic & $\mathrm{Th}$ & + & + & - & - \\
\hline 140 & Cestrum nocturnum L. & Solanaceae & Rat ke rani & Herb & Mic & Cha & + & + & + & + \\
\hline 141 & Uritica dioca $\mathrm{L}$. & Urticaceae & Unknown & Herb & Mic & Th & + & + & - & + \\
\hline 142 & Verbena officinalis L. & Verbinaceae & Unknown & Herb & Mic & Th & + & + & + & + \\
\hline 143 & Vitis vinifera L. & Vitaceae & Angur & $\begin{array}{c}\text { Root } \\
\text { climber }\end{array}$ & Mes & $\mathrm{Mp}$ & + & + & + & + \\
\hline 144 & Aloe vera (L.) Burm.f,Fl. & Xanthorrhoeaceae & Alovera & Herb & Mes & Cha & + & + & + & + \\
\hline 145 & Cucuma longa L. & Zingiberaceae & Korkaman & Herb & Mes & Geo & + & + & + & + \\
\hline 146 & Peganum harmala L. & Zygophyllaceae & Spelany & Shrub & Mic & Cha & + & - & - & - \\
\hline
\end{tabular}

Note: Lep = Leptophyll, Nan = Nanophyll, Mic = Microphyll, Mes =Mesophyll, Mac = Macrophyll, Meg = Megaphyll, Ap = Aphyllus Th = Therophytes, Geo $=$ Geophytes, Cha =Chaemophytes, Hem = Hemicryptophytes, Nan = Nanophanerophytes, Micp = Microphanerophyts, Mesp = Mesophanerophytes, Megp = Megaphanerophyytes, $\mathrm{P}=$ Parasite, $\mathrm{Sp}=$ Spring, $\mathrm{Sm}=$ Summer, $\mathrm{W}=$ Winter, $\mathrm{Au}=$ Autumn 


\section{Conclusion}

From the present study it was concluded that flora of the area is diverse and consisted of 146 plants species distributed among 58 families and 127 genera. The dominant life form was therophytes with 66 species (45.20\%) followed by Microphanerophytes having 22 species $(15.06 \%)$.While, microphyll dominating the leaf size spectra. In district Charsadda harsh winter creates unfavourable conditions which resulted in the abundance of these life forms. The locality also confronting a number of threats in the form of overgrazing, soil erosion, construction and agricultural extension. These factors combined with anthropogenic pressures are a serious threat to local biodiversity. Therefore, proper conservation strategies are the need of the hour to conserve this natural wealth for the generation to come.

\section{Authors' contributions}

Conceived and designed the experiments: $S$ Shah \& S Ullah, Performed the experiments: S Shah, Analyzed the data: Y Khan \& T Yaseen, Contributed reagents/ materials/ analysis tools: S Ullah, MF Basit, Wrote \& revised the paper: Y Khan \& T Yaseen.

\section{References}

1. Keith DA (1988). Floristic lists of New South Wales (III). Cunninghamia 2(1): 39-73.

2. Ali SI (2008). The significance of flora with special reference to Pakistan. Pak J Bot 40(30): 967-971.

3. Khan SM \& Ahmad H (2014). Role of Indigenous Arqiyat distillery in conservation of Rosa species. Int $J$ of Phytomedicine 6(2): 162.

4. Akbarinia MO, Zare H, Hoseini SM \& Ejtehadi H (2004). Study on vegetation structure, floristic composition and chorology of silver birch communities at Sangdeh, forest of Hyrcanian region. In Natural Resources.

5. Ali SI (2008). Significance of flora with special reference to Pakistan. Pak J Bot 40(3): 967-971.

6. Stace CA (1989). Plant taxonomy and biosystematics. Edvard Arnold, London.
7. Sarmiento G \& Monasterio M (1983). Life forms and phenology. Ecosystems of the World. 13:79-108.

8. Raunkiaer C (1934). The life form at plants and statistical geography. Darendon Press, Oxford 23.

9. Anonymous (1998). District Census Report of Charsadda, PCO, Govt. of Pakistan.

10. Khan S, Shahnaz M, Jehan N, Rehman S, Shah MT \& Din I (2013). Drinking water quality and human health risk in Charsadda district, Pakistan. J Clean Prod 60: 93-101.

11. Nasir E \& Ali SI (1970-1989). Flora of Pakistan, Islamabad, Karachi.

12. Anonymous (2018). Population and household detail from block to district level: Khyber Pakhtunkhwa, Govt. of Pakistan.

13. Ali SI \& Nasir YJ (1991-1993). Flora of Pakistan Nos. 191-193. Department of Botany, Karachi University, Karachi Pakistan.

14. Ali SI \& Qaiser M (1993-2018). Flora of Pakistan, Department of Botany, University of Karachi.

15. Noor A, Anwar H, Sidra A \& Shumaila B (2013). Accumulation of heavy metals in edible parts of vegetables irrigated with waste water and their daily intake to adults and children, district Mardan, Pakistan. Food Chem 136: 1515-1523.

16. Jan RU, Khan RU, Rehman HU, Khan AZ, Waheed MA \& Khan IU (2016). Ethnobotanically important flora of Tehsil Tangi, District Charsadda. Pak J Pham Sci 8(3): 108-116.

17. Khan MN, Hadi F, Razaq A \& Shah SM (2017). Utilitarian aspects of weeds and their ecological characteristics in Ochawala valley, District Charsadda, Pakistan. APRN J Agric and Biol Sci 12(5): 182-189.

18. Ali SI \& Qaiser M (1993-2012). Flora of Pakistan. Nos. 194-221. Department of Botany, Karachi University, Karachi Pakistan.

19. Qureshi MA \& Khan SA (1965-67). Flora of Peshawar District and Khyber Agency. Pak J For 15: 364-393.

20. Qureshi MA \& Khan KA (1971). An illustrated Flora of Peshawar District and Khyber Agency. Ranunculaceae to Moringaceae. Pak J For 1: 212. 
21. Khan M, Hussain F \& Musharaf S (2013). Floristic composition and biological characteristics of the vegetation of Sheikh Maltoon Town District Mardan, Pakistan. Annu Res Rev 6: 31-41.

22. Khan M, Hussain F \& Musharaf S (2014). Floristic Composition and Ecological Characteristics of Shahbaz Garhi, District Mardan, Pakistan. GJSFR 1: 17.

23. Amjad MS, Arshad M, Sadaf HM, Akrim F \& Arshad A (2016). Floristic composition, biological spectrum and conservation status of the vegetation in Nikyal valley, Azad Jammu and Kashmir. Asian Pac J Trop Dis 6(1): 63-9.

24. Ilyas M, Qureshi R, Arshad M \& Mirza SN (2013). A Preliminary checklist of the vascular flora of Kabal Valley, Swat, Pakistan. Pak J Bot 45(2): 605-615.

25. Hussain F, Shah SM, Badshah L \& Durrani MJ (2015). Diversity and ecological characteristics of flora of mastuj Valley, District Chitral, Hindukush Range, Pakistan. Pak J Bot 47(2): 495510.

26. Badshah L, Hussain F \& Sher Z (2013). Floristic inventory, ecological characteristics and biological spectrum of rangeland, District Tank, Pakistan. Pak J Bot 45(4): 1159-1168.

27. Durrani MJ, Razaq A, Muhammad SG \& Hussain F (2010). Floristic diversity, ecological, characteristics and ethnobotonical profile of plants of Aghbergrange lands, Balochistan, Pakistan. Pak J Pl Sci 16(1): 29-36.

28. Cain SA (1950). Life-forms and phytoclimate. Bot Res 16(1): 21-32.

29. Cain SA \& Castro GM (1959). Manual of vegetation analysis Harper \& Brother Publ. New York, 325.

30. Asri Y (1999). Ecological study of arid zone plant communities (Case study: biosphere reservoir, province). Ph.D. Thesis. Islamic Azad University, Science and Research Campus.

31. Saxina AK, Pandey TP \& Singh JS (1987). Altitudinal variation in the vegetation of Kaumaun Himalaya. Perspective in Environ Bot 44-66.

32. Al-Sherif EA, Ayesh AM \& Rawi SM (2013). Floristic composition, life form and chorology of plant life at Khulais region, Western Saudi Arabia. Pak J Bot 45(1): 29-38.

33. Wariss HM, Mukhtar M, Anjum S, Bhatti GR, Pirzada SA \& Alam K (2013). Floristic composition of the plants of the Cholistan Desert, Pakistan. Am J Plant Sci 412-419.

34. Sher Z, Hussain F \& Badshah L (2014). Biodiversity and ecological characterization of the flora of Gadoon rangeland, district Swabi, Khyber Pukhtunkhwa, Pakistan. Iran J Bot 20(1): 96-108.

35. Ali S, Shuaib M, Ali H, Ullah S, Ali K \& Hussain S (2017). Floristic list and their ecological characteristics, of plants at village Sherpao District Charsadda, KPPakistan. J of Med P 5(5): 295-309.

36. Seraj SS, Jrais RN \& Ayyad SK (2014).Floristic composition, life form and chorology of plant life at Al-Saoda, Asir Region, South-Western Saudi Arabia. $J$ Biol Agr \& Hc 4(26): 60-68.

37. Salama FM, Sayed SA \& Abd el AA (2014). Plant communities and floristic composition of the vegetation of Wadi AlAssiuty and Wadi Habib in the Eastern Desert, Egypt. Not Sci Biol 6(2): 196-206.

38. Ali A, Badshah L, Hussain F \& Shinwari ZK (2016). Floristic composition and ecological characteristics of plants of chail valley, district Swat, Pakistan. Pak J Bot 48(3): 1013-1026.

39. Oosting HJ (1956). The Study of Plant Communities, WH Freeman and Co., Sanfrancisco, 2nd edition, pp 69-78.

40. Ullah S \& Badshah L (2017). Floristic structure and ecological attributes of Jelar valley flora, district Upper Dir, Pakistan. JBES 10(5): 89-105.

41. Khan M, Hussain F \& Musharaf S (2012). Biological characteristics of plant species in Tehsil Takht-e-Nasrati Pakistan. J Biol Environ Sci 2(3): 42-47.

42. Malik ZH, Hussain F \& Malik NZ (2007). Life form and leaf size spectra of plant communities Harbouring Ganga Chotti and Bedori Hills during 1999-2000. Int J Agric Biol 9(6): 833-848. 